MATHEMATICS OF COMPUTATION

Volume 77, Number 261, January 2008, Pages 21-39

S 0025-5718(07)02015-7

Article electronically published on May 14, 2007

\title{
UNIFORM ERROR ESTIMATES IN THE FINITE ELEMENT METHOD FOR A SINGULARLY PERTURBED REACTION-DIFFUSION PROBLEM
}

\author{
DMITRIY LEYKEKHMAN
}

\begin{abstract}
Consider the problem $-\epsilon^{2} \Delta u+u=f$ with homogeneous Neumann boundary condition in a bounded smooth domain in $\mathbb{R}^{N}$. The whole range $0<\epsilon \leq 1$ is treated. The Galerkin finite element method is used on a globally quasi-uniform mesh of size $h$; the mesh is fixed and independent of $\epsilon$.

A precise analysis of how the error at each point depends on $h$ and $\epsilon$ is presented. As an application, first order error estimates in $h$, which are uniform with respect to $\epsilon$, are given.
\end{abstract}

\section{INTRODUCTION}

Consider the following problem: find a function $u(x, \varepsilon)$ that satisfies the following partial differential equation with homogeneous Neumann boundary conditions:

$$
\begin{aligned}
-\varepsilon^{2} \Delta u+u & =f(x, \varepsilon) & & \text { in } \Omega, \\
\frac{\partial u}{\partial n} & =0 & & \text { on } \partial \Omega,
\end{aligned}
$$

where $\Omega$ is a smooth bounded domain in $\mathbb{R}^{N}, N \geq 2$. Here $\varepsilon$ is a parameter, $0<\varepsilon \leq 1$, and $f(x, \varepsilon)$ is a uniformly bounded function in $L_{2}(\Omega)$.

In this paper we consider the whole range $0<\varepsilon \leq 1$. In contrast to many other investigations (cf. below), the mesh is not allowed to vary with $\varepsilon$. We assume that the mesh is globally quasi-uniform, not necessarily regular, of size $h$. When $\varepsilon$ is of order one, the problem is uniformly elliptic, the solution $u$ is "well behaved", and the precise theory of A.H. Schatz [7] explains in detail how the error behaves (cf. below in this introduction). On the other hand, when $\varepsilon$ approaches zero, the problem becomes singularly perturbed, and the solution may develop boundary layers. These boundary layers are somewhat less pronounced in our case of Neumann boundary conditions than in the case of Dirichlet boundary conditions. Hence, in our investigation with Neumann conditions, we can establish first order convergence in $h$, uniformly in $\varepsilon$, with a mesh independent of $\varepsilon$.

To achieve first order convergence in the Dirichlet case, or, to achieve higher order convergence than first in the Neumann case, will require remeshing according to each $\varepsilon$. In practice, this is rather undesirable if one wants to solve a number of problems (1.1) with varying $\varepsilon$.

Received by the editor June 8, 2005 and, in revised form, November 18, 2006

2000 Mathematics Subject Classification. Primary 65N30.

Key words and phrases. Finite element, singularly perturbed, pointwise estimates, reactiondiffusion.

(c) 2007 American Mathematical Society Reverts to public domain 28 years from publication 
A great amount of research has been done on numerical methods for singularly perturbed reaction-diffusion problems. Most of the work has been focused on the problems either in one space dimension or on very special domains in the plane. For instance, in a recent paper [2, the authors considered the problem with Dirichlet boundary conditions on a unit square and proved second order convergence in $h$ uniformly in $\varepsilon$ for the standard central finite difference method with mesh refinement depending on $\varepsilon$.

Results for general domains in $\mathbb{R}^{N}, N \geq 2$, are rare, especially in the maximum norm. Two such results we would like to mention are [1] and [5], where the problem was considered on a general smooth plane domain with Dirichlet boundary conditions. In those papers, with special meshes depending on $\varepsilon$, the authors obtained a second order estimate in the maximum norm over the whole domain, including the boundary layer, uniformly in $\varepsilon$. Furthermore, as in [2, the degrees of freedom of the used spaces are bounded by $C h^{-2}$ uniformly in $\varepsilon$.

The aim of this paper is somewhat different. We consider the standard Galerkin finite element method on a globally quasi-uniform mesh of size $h$. The mesh is independent of $\varepsilon$. The Galerkin finite element solution $u_{h} \in S_{h}^{r}$ satisfies

$$
\varepsilon^{2}\left(\nabla u_{h}, \nabla \chi\right)+\left(u_{h}, \chi\right)=(f, \chi), \text { for all } \chi \in S_{h}^{r},
$$

where $(v, w)$ denotes the $L_{2}(\Omega)$ inner product $\int_{\Omega} v(x) w(x) d x$. The precise definition of $S_{h}^{r}$ is given in Chapter 2. For now, we may think of $S_{h}^{r}$ as a set of continuous piecewise polynomials of total degree $r-1$ on globally quasi-uniform partitions of $\Omega$.

Instead of deriving an " $\varepsilon$-specific" method that guarantees a certain order of convergence uniformly in $\varepsilon$, we give a precise analysis of how the error between the real solution $u$ and the Galerkin solution $u_{h}$ at each point depends on $h$ and $\varepsilon$. Then as an application of our main result, we show that the error is of first order in $h$, uniformly in $\varepsilon$.

Before we describe the main result, let us review pointwise error estimates in two extreme cases, $\varepsilon=0$ and $\varepsilon=1$.

When $\varepsilon=0$, problem (1.2) degenerates formally into the zero order equation

$$
\left(u_{h}, \chi\right)=(f, \chi)
$$

i.e. $u_{h}$ is the $L_{2}$ projection onto $S_{h}^{r}$. Pointwise behavior of $L_{2}$ projections are well analyzed (cf. Chapter 7 in [13]), and it can be shown that the error satisfies

$$
\left|\left(u-u_{h}\right)(x)\right| \leq C \min _{\chi \in S_{h}^{r}}\left\|e^{-c \frac{|x-y|}{h}}(u-\chi)(y)\right\|_{L_{\infty}(\Omega)},
$$

for some positive constants $c$ and $C$ independent of $u, u_{h}, x$, and $h$.

When $\varepsilon=1$, the equation (1.2) is uniformly elliptic and sharp pointwise error estimates were obtained by A.H. Schatz in [7]. To describe his main result we need to introduce some notation. Fix $x \in \Omega$ and consider the weight

$$
\sigma(y)=\sigma_{h, x}(y)=\frac{h}{h+|x-y|}, \text { for } y \in \mathbb{R}^{N} .
$$

Notice that $\sigma(y)=O(1)$ if $|x-y|=O(h)$ and $\sigma(y)=O(h)$ if $|x-y|=O(1)$.

For $1 \leq p \leq \infty$, a real number $s$, and a fixed $x$, we define the weighted norms over domains $\Omega$ by

$$
\|u\|_{L_{p}(\Omega), \sigma, s}=\left\|\sigma_{h, x}^{s}(y) u(y)\right\|_{L_{p}(\Omega)} .
$$


The main result of [7] says that, for any $0 \leq s \leq r-2$,

$$
\left|\left(u-u_{h}\right)(x)\right| \leq C \ell_{h} \min _{\chi \in S_{h}^{r}}\|u-\chi\|_{L_{\infty}(\Omega), \sigma, s},
$$

where the constant $C$ is independent of $u, u_{h}, h$, and $x$, and the logarithmic term $\ell_{h}=|\log h|$ is necessary only when $s=r-2$.

The main result in this paper can be thought of as an interpolation between these two extreme cases and may roughly be stated as follows: Let $0<\varepsilon \leq 1$. Then, for any fixed $x \in \Omega$ and $0 \leq s \leq r-2$,

$$
\left|\left(u-u_{h}\right)(x)\right| \leq C \ell_{h} \min _{\chi \in S_{h}^{r}}\left\|e^{-c \frac{|x-y|}{\varepsilon+h}}(u-\chi)(y)\right\|_{L_{\infty}(\Omega), \sigma, s},
$$

where $C$ and $c$ are independent of $u, u_{h}, h, \varepsilon$, and $x$, and the logarithmic term $\ell_{h}=|\log h|$ is necessary only when $s=r-2$ and $\varepsilon \gg h$.

From (1.7) it is easy to see that if $\varepsilon=O(h)$, then $u_{h}$ behaves essentially like the $L_{2}$ projection, and if $\varepsilon=O(1)$, we get the A.H. Schatz's weighted result (1.6).

The estimate (1.7) is useful for analyzing singularly perturbed problems, i.e. when $\varepsilon$ is small. We now give some applications.

For the rest of the introduction we assume that $\varepsilon$ is small, for example $\varepsilon=O\left(h^{\alpha}\right)$, for some $\alpha>0$.

Let $B_{d}$ denote a ball of radius $d$ centered at $x$. From (1.7), taking into consideration only the exponential weight, we have

$$
\left|\left(u-u_{h}\right)(x)\right| \leq C \ell_{h} \min _{\chi \in S_{h}^{r}}\|u-\chi\|_{L_{\infty}\left(B_{d}\right)}+C \ell_{h} e^{-\frac{c d}{\varepsilon+h}}\|u\|_{L_{\infty}\left(\Omega \backslash B_{d}\right)} .
$$

If $u \in W_{\infty}^{r}\left(B_{d}\right), u \in L_{\infty}\left(\Omega \backslash B_{d}\right)$, and $d>\kappa(\varepsilon+h)|\log h|$, for $\kappa$ sufficiently large, then $\left|\left(u-u_{h}\right)(x)\right| \leq C \ell_{h} h^{r}$. Thus we can conclude that Galerkin solution $u_{h}$ approximates $u$ to the optimal order on subdomains where the solution $u$ is sufficiently smooth.

On the other hand, in the boundary layer we have to be careful since the derivatives of $u$ may depend on $\varepsilon$. In Corollary 2.3 we show, assuming $f \in W_{\infty}^{1}(\Omega)$, that, for any $x \in \Omega$, there exists a positive constant $C$ independent of $\varepsilon$ and $h$, such that

$$
\left|\left(u-u_{h}\right)(x)\right| \leq C|\log h|^{3} \min \left\{h^{2} / \varepsilon, h\right\}\|f\|_{W_{\infty}^{1}(\Omega)} .
$$

Therefore, we may conclude that the Galerkin approximation for the Neumann problem is of almost first order uniformly in $\varepsilon$ in the global maximum norm, provided $\|f\|_{W_{\infty}^{1}}$ is uniformly bounded in $\varepsilon$.

One way to increase the order of convergence in the boundary layer is by using matched asymptotic expansion (cf. [4). For example, let $x^{\prime} \in \partial \Omega$ denote the point where the normal from $x$ meets the $\partial \Omega$. Set

$$
u_{\varepsilon}(x)=f(x)+\frac{\partial f}{\partial n}\left(x^{\prime}\right) e^{-\frac{\left|x-x^{\prime}\right|}{\varepsilon}},
$$

where $f$ is evaluated at $\varepsilon=0$. The first term on the right is called the "regular inner expansion" and the second term is the "boundary layer correction". It is not hard to show that in the boundary layer $\left\|u-u_{\varepsilon}\right\|_{L_{\infty}} \leq C \varepsilon^{2}$. Thus in the boundary layer, switching from the Galerkin approximation $u_{h}$ to the matched expansion $u_{\varepsilon}$ when $\varepsilon<O\left(h^{2 / 3}\right)$, gives a "method" of uniform order almost $4 / 3$ in the global maximum norm. Of course if more terms in the matched asymptotic expansion are available we can increase the order, but in general they are much harder to compute. 
Remark 1. Using the same techniques we can prove a similar result for the above problem with Dirichlet boundary conditions on convex bounded domains in $\mathbb{R}^{N}$ for piecewise linear finite element spaces.

In the case of Dirichlet boundary conditions, the boundary layer is more pronounced, and under the same basic assumptions using similar techniques we can only show

$$
\left|\left(u-u_{h}\right)(x)\right| \leq C \ell_{h} \min \left\{h^{2} / \varepsilon^{2}, 1\right\}\|f\|_{L_{\infty}} .
$$

The matched asymptotic expansion in the Dirichlet case is

$$
u_{\varepsilon}(x)=f(x)-f\left(x^{\prime}\right) e^{-\frac{\left|x-x^{\prime}\right|}{\varepsilon}},
$$

and on the boundary layer we have $\left\|u-u_{\varepsilon}\right\|_{L_{\infty}} \leq C \varepsilon$. Thus switching from the Galerkin solution $u_{h}$ to the matched expansion $u_{\varepsilon}$ in the boundary layer when $\varepsilon<O\left(h^{2 / 3}\right)$ gives a method of uniform order only $2 / 3$ in the global maximum norm.

This work is based on the paper [11 by A.H. Schatz and L.B. Wahlbin, in which the authors showed a somewhat similar result restricted to the piecewise linear case $r=2$ and space dimension $N=2$. This paper sharpens the above result and removes the restrictions on the dimension and the order of the finite element spaces in the case when $a \equiv 1$.

The proof of our main result (1.7) is based on a Green's function estimate for the continuous problem, which is obtained from a Green's function estimate for the parabolic problems [3], and local energy estimates for the approximate Green's function. An essential analytical tool for the derivation of (1.7) is a "kick-back" argument, which was developed by A.H. Schatz and L.B. Wahlbin and was used in a number of papers, for example [8, 9], [10].

Outline of the paper. Section 2 contains the assumptions on the finite element spaces, the statement of the main result, and Corollary 2.3 with a proof. Sections 3.4 are preliminary and contain global and local energy estimates, which are used in the proof of the main result. In Sections 5 6 we prove the main result. Finally, in the Appendix we prove Lemma 2.2, the pointwise estimate of the Green's function for the continuous problem.

\section{Preliminaries AND Statement of the MAin Result}

With $0<h<1 / 2$ a parameter, let $\tau_{j}^{h}, j=1, \ldots, J_{h}$, be disjoint open sets, elements, which form a partition of $\Omega$ and fit the boundary exactly, i.e. $\bar{\Omega}=$ $\bigcup_{j=1}^{J_{h}} \bar{\tau}_{j}^{h}$. For each such partition, let $S_{h}^{r}=S_{h}^{r}(\Omega) \subset W_{\infty}^{1}(\Omega)$ be a finite-dimensional space. We will use $W_{p}^{l}(D)$, with $1 \leq p \leq \infty, l=0,1, \ldots$, and a set $D$ to denote the standard Sobolev spaces with $\|\cdot\|_{W_{p}^{l}(D)}$ and $|\cdot|_{W_{p}^{l}(D)}$ their norms and semi-norms respectively. When needed, we will also use the piecewise norms

$$
\|u\|_{W_{p}^{l}(D)}^{(h)}=\left(\sum_{\tau_{j}^{h} \cap D \neq \emptyset}\|u\|_{W_{p}^{l}\left(\tau_{j}^{h} \cap D\right)}^{p}\right)^{1 / p} .
$$

Similarly, we have the weighted piecewise norms

$$
\|u\|_{W_{p}^{l}(D), \sigma, s}^{(h)}=\sum_{0 \leq|\alpha| \leq l}\left\|\sigma^{s} D_{x}^{\alpha} u\right\|_{L_{p}(D)}^{(h)} .
$$


Next, we will state some standard assumptions about finite element spaces. Assume there exist positive constants $\delta, k, \underline{k}, \bar{k}, C_{1}, C_{2}, C_{3}, C_{4}$, and an integer $r \geq 2$, all independent of $h$, such that the assumptions 2.1 through 2.4 below hold.

The first assumption expresses the global quasi-uniformity of the partition of $\Omega$ and a trace inequality at the boundary of each element.

2.1. Quasi-uniformity and trace. (i) Each $\tau_{j}^{h}$ contains a ball of radius $\underline{k} h$ and is contained in a ball of radius $\bar{k} h$.

(ii) For $0<h<\frac{1}{2}$ and $j=1,2, \ldots, J_{h}$,

$$
\int_{\partial \tau_{j}^{h}}|\nabla v| d S_{j} \leq C_{1}\left(h^{-1}|v|_{W_{1}^{1}\left(\tau_{j}^{h}\right)}+|v|_{W_{1}^{2}\left(\tau_{j}^{h}\right)}\right), \forall v \in W_{1}^{2}\left(\tau_{j}^{h}\right) .
$$

The second assumption is a standard inverse property. For $D \subset \Omega, S_{h}^{r}(D)$ will denote the restriction of $S_{h}^{r}$ to $D$.

2.2. Inverse property. Let $\chi \in S_{h}^{r}(D)$, where $D$ is any union of closures of elements. Then for $0 \leq k \leq l \leq 2,1 \leq q \leq p \leq \infty$,

$$
\|\chi\|_{W_{p}^{l}(D)}^{(h)} \leq C_{2} h^{-(l-k)-N\left(\frac{1}{q}-\frac{1}{p}\right)}\|\chi\|_{W_{q}^{k}(D)}^{(h)} .
$$

Our third assumption is about local approximation properties of the finite element spaces. For $D$ a subset of $\Omega$ we let $D_{d}=\{x \in \Omega: \operatorname{dist}(x, D) \leq d\}$.

2.3. Local approximation. Let $d \geq k h$. There exists a linear operator $I_{h}$ : $W_{1}^{1}(\Omega) \rightarrow S_{h}^{r}(\Omega)$ such that for any $D$ the following holds:

$$
\left\|v-I_{h} v\right\|_{W_{p}^{s}(D)}^{(h)} \leq C_{3} h^{l-s}\|v\|_{W_{p}^{l}\left(D_{d}\right)}, \text { for } 0 \leq s \leq l \leq r, 1 \leq p \leq \infty .
$$

2.4. Superapproximation. If the function to be approximated is of a certain special form, we have an assumption known as superapproximation.

Let $d \geq k h$ and $\omega \in C_{0}^{\delta}\left(\bar{D}_{2 d}\right)$; then for any $\psi \in S_{h}^{r}\left(D_{3 d}\right)$ there exists $\eta \in S_{h}^{r}\left(D_{3 d}\right)$, vanishing outside of $D_{3 d}$ such that

$$
\|\omega \psi-\eta\|_{W_{2}^{l}\left(D_{3 d}\right)} \leq C_{4} h\|\omega\|_{W_{\infty}^{\delta}\left(D_{2 d}\right)}\|\psi\|_{W_{2}^{l}\left(D_{3 d}\right)}, \quad l=0,1 .
$$

Furthermore, if $\omega \equiv 1$ on $D_{d}$, then $\eta=\psi$ on $D$, and the last factor may be replaced by $\|\psi\|_{W_{2}^{l}\left(D_{3 d} \backslash D\right)}$.

We can now state our main result, which expresses how the error at a point depends on the continuous solution.

Theorem 2.1. Suppose that assumptions 2.1 through 2.4 hold and $u$ and $u_{h} \in S_{h}^{r}$ satisfy (1.1) and (1.2) respectively. Let $x \in \Omega, 0<\varepsilon \leq 1$, and let s satisfy $0 \leq s \leq r-2$, for $r \geq 2$. Furthermore assume $1-\varepsilon c_{2}>0$, where $c_{2}$ is the smallest real number such that the estimate in Lemma 7.1 holds. Then there exist constants $C$ and $c$ independent of $x, u, u_{h}, \varepsilon$, and $h$ such that

$$
\left|\left(u-u_{h}\right)(x)\right| \leq C \ell_{h} \min _{\chi \in S_{h}^{r}}|| e^{-c \frac{|x-y|}{\varepsilon+h}}(u-\chi)(y) \|_{L_{\infty}(\Omega), \sigma, s},
$$

where $\ell_{h}=1$, if $s<r-2$ or $\varepsilon=O(h)$ and $\ell_{h}=|\log h|$, if $s=r-2$ and $\varepsilon \gg h$.

Remark 2. If $\varepsilon=O(h)$, then the exponential weight is the dominating one and we have

$$
\left|\left(u-u_{h}\right)(x)\right| \leq C \min _{\chi \in S_{h}^{r}}\left\|e^{-c \frac{|x-y|}{h}}(u-\chi)(y)\right\|_{L_{\infty}(\Omega)},
$$

i.e. $u_{h}$ behaves like the $L_{2}$ projection. 
The major tool in obtaining the main result is the following estimate for the Green's function of the continuous problem (1.1).

Lemma 2.2. The solution of (1.1) may be represented in terms of the Green's function $K^{\varepsilon}(x, y)$, for $x, y \in \Omega$, as

$$
u(x)=\int_{\Omega} K^{\varepsilon}(x, y) f(y) d y .
$$

Assume that the boundary $\partial \Omega$ is sufficiently smooth and $1-\varepsilon c_{2}>0$, where $c_{2}$ is the smallest real number such that the estimate in Lemma 7.1 holds. Then for any multi-integer $m$, there exist constants $C$ and $c_{0}>0$ such that for the Green's function $K^{\varepsilon}(x, y), x, y \in \Omega$, we have

$$
\left|D_{x}^{m} K^{\varepsilon}(x, y)\right| \leq \frac{C e^{-c_{0} \frac{|x-y|}{\varepsilon}}}{\varepsilon^{N+|m|}} \times \begin{cases}1, & \text { if } N+|m|=1, \\ 1+\left|\log \frac{|x-y|}{\varepsilon}\right|, & \text { if } N+|m|=2, \\ \left(\frac{|x-y|}{\varepsilon}\right)^{2-N-|m|}, & \text { if } N+|m| \geq 3 .\end{cases}
$$

The proof of this result is given in the Appendix. It is based on [3].

Remark 3. If $\varepsilon=O(1)$, then the above estimate reduces to the well known estimate for the Green's function for the uniformly elliptic problem (cf. Krasovski [6]).

Corollary 2.3. Under the assumptions of Theorem 2.1 and assuming $S_{h}^{r} \subset C(\bar{\Omega})$ and $f \in W_{\infty}^{r}(\Omega)$, we have for any $1 \leq s \leq r$

$$
\left|\left(u-u_{h}\right)(x)\right| \leq C \ell_{h} \cdot \min \left\{\begin{array}{l}
\frac{h^{2}|\log h| \log \left(\frac{1}{\varepsilon}\right)}{\varepsilon}\|f\|_{W_{\infty}^{1}(\Omega)}, \\
\varepsilon|\log h|^{2} \log \left(\frac{1}{\varepsilon}\right)\|f\|_{W_{\infty}^{1}(\Omega)}+h^{s}\|f\|_{W_{\infty}^{s}(\Omega)} .
\end{array}\right.
$$

Proof. Since $S_{h}^{r} \subset C(\bar{\Omega})$, the standard interpolant satisfies (cf. [12 Section 4)

$$
\left\|u-I_{h} u\right\|_{L_{\infty}(\Omega)} \leq C|\log h| h^{2}\|\Delta u\|_{L_{\infty}(\Omega)} .
$$

From Theorem 2.1 we have

$$
\left|\left(u-u_{h}\right)(x)\right| \leq C \ell_{h} \min _{\chi \in S_{h}^{r}}\|u-\chi\|_{L_{\infty}(\Omega)} \leq C \ell_{h}|\log h| h^{2}\|\Delta u\|_{L_{\infty}(\Omega)} .
$$

The top part of estimate (2.3) will follow from (2.4) and the following lemma.

Lemma 2.4. There exists a constant $C$ independent of $\varepsilon$ such that

$$
\|\Delta u\|_{L_{\infty}(\Omega)} \leq \frac{C}{\varepsilon} \log \left(\frac{1}{\varepsilon}\right)\|f\|_{W_{\infty}^{1}(\Omega)} .
$$

Proof. Since the case $\varepsilon>1 / 2$ is easy, we assume $\varepsilon \leq 1 / 2$. Assuming that $u$ and $f$ are sufficiently smooth, we have

$$
\|\Delta u\|_{L_{\infty}(\Omega)}=\frac{1}{\varepsilon^{2}}\|u-f\|_{L_{\infty}(\Omega)} .
$$

For $x \in \Omega$,

$$
u(x)-f(x)=\int_{\Omega} K^{\varepsilon}(x, y) f(y) d y-f(x)=\int_{\Omega} K^{\varepsilon}(x, y)(f(y)-f(x)) d y,
$$

where we used that $\int_{\Omega} K^{\varepsilon}(x, y) d y=1$ for any $x$ since the function $v \equiv 1$ solves

$$
\begin{aligned}
-\varepsilon^{2} \Delta u+u=1 & & \text { in } \Omega, \\
\frac{\partial u}{\partial n}=0 & & \text { on } \partial \Omega .
\end{aligned}
$$


Thus,

$$
\begin{aligned}
u(x)-f(x) & =\int_{\Omega \backslash B_{d}} K^{\varepsilon}(x, y)(f(y)-f(x)) d y+\int_{B_{d} \cap \Omega} K^{\varepsilon}(x, y)(f(y)-f(x)) d y \\
& =J_{1}+J_{2},
\end{aligned}
$$

where $B_{d}$ denotes a ball centered at $x$ of radius $d$. Choose $d=\kappa \varepsilon \log \left(\frac{1}{\varepsilon}\right)$, with $\kappa$ sufficiently large. Using the estimates of Lemma 2.2 in the case $N \geq 3$, we have

$$
\left|J_{1}\right| \leq C\|f\|_{L_{\infty}(\Omega)} \frac{1}{\varepsilon^{N}} e^{-c_{0} \kappa \log \left(\frac{1}{\varepsilon}\right)} \leq C \varepsilon\|f\|_{L_{\infty}(\Omega)},
$$

provided $c_{0} \kappa \geq N+1$.

By the Mean Value Theorem we can bound $J_{2}$ by

$$
\left|J_{2}\right| \leq C \kappa \log \left(\frac{1}{\varepsilon}\right) \varepsilon\|f\|_{W_{\infty}^{1}\left(B_{d}\right)} \int_{\Omega}\left|K^{\varepsilon}(x, y)\right| d y .
$$

It remains to show that $\int_{\Omega}\left|K^{\varepsilon}(x, y)\right| d y \leq C$. Using Lemma 2.2 with $N \geq 3$,

$$
\int_{\Omega}\left|K^{\varepsilon}(x, y)\right| d y \leq C \int_{\Omega} e^{-c_{0} \frac{|x-y|}{\varepsilon}} \frac{d y}{\varepsilon^{2}|x-y|^{N-2}} .
$$

Switching to polar coordinates, $|x-y|=\rho, d y=C \rho^{N-1} d \rho$, we have

$$
\int_{\Omega}\left|K^{\varepsilon}(x, y)\right| d y \leq C \int_{0}^{R} e^{-c_{0} \frac{\rho}{\varepsilon}} \frac{\rho}{\varepsilon} \frac{d \rho}{\varepsilon} \leq C .
$$

Thus we have the first estimate of the corollary in the case $N \geq 3$. The case $N=2$ is very similar.

To show the other part of estimate (2.3), we notice that

$$
u-u_{h}=\varepsilon^{2} \Delta u+f-\varepsilon^{2} \Delta_{h} u_{h}-P_{h} f,
$$

where $P_{h}: L_{2}(\Omega) \rightarrow S_{h}^{r}$ is the $L_{2}$ projection defined by

$$
\left(P_{h} v, \chi\right)=(v, \chi) \text {, for } \chi \in S_{h}^{r},
$$

and $\Delta_{h}: S_{h}^{r} \rightarrow S_{h}^{r}$ is the discrete Laplacian defined by

$$
-\left(\Delta_{h} v, \chi\right)=(\nabla v, \nabla \chi) \text {, for } \chi \in S_{h}^{r} .
$$

Using the triangle inequality we have

$$
\left\|u-u_{h}\right\|_{L_{\infty}(\Omega)} \leq \varepsilon^{2}\left\|\Delta u-\Delta_{h} u_{h}\right\|_{L_{\infty}(\Omega)}+\left\|f-P_{h} f\right\|_{L_{\infty}(\Omega)} .
$$

Using the approximation properties of the $L_{2}$ projection we can bound the second term as

$$
\left\|f-P_{h} f\right\|_{L_{\infty}(\Omega)} \leq C h^{s}\|f\|_{W_{\infty}^{s}(\Omega)}, \text { for any } 0 \leq s \leq r .
$$

For the first term on the right hand side in (2.9) by the triangle inequality, we have

$$
\left\|\Delta u-\Delta_{h} u\right\|_{L_{\infty}(\Omega)} \leq\left\|\Delta u-\Delta_{h} R_{h} u\right\|_{L_{\infty}(\Omega)}+\left\|\Delta_{h} R_{h} u-\Delta_{h} u_{h}\right\|_{L_{\infty}(\Omega)},
$$

where $R_{h}: H^{1}(\Omega) \rightarrow S_{h}^{r}$ is the Ritz projection defined by

$$
\left(\nabla R_{h} v, \nabla \chi\right)=(\nabla v, \nabla \chi), \text { for } \chi \in S_{h}^{r} .
$$


DMITRIY LEYKEKHMAN

Using the operator identity $\Delta_{h} R_{h}=P_{h} \Delta$, the stability of the $L_{2}$ projection in $L_{\infty}$ norm, and (2.5), we can bound the first term on the right hand side of (2.11) as

$$
\begin{aligned}
\left\|\Delta u-\Delta_{h} R_{h} u\right\|_{L_{\infty}(\Omega)} & =\left\|\Delta u-P_{h} \Delta u\right\|_{L_{\infty}(\Omega)} \\
& \leq C\|\Delta u\|_{L_{\infty}(\Omega)} \leq \frac{C}{\varepsilon} \log \left(\frac{1}{\varepsilon}\right)\|f\|_{W_{\infty}^{1}(\Omega)} .
\end{aligned}
$$

Applying the inverse inequality and the triangle inequality on the second term on the right hand side of (2.11), we have

$$
\left\|\Delta_{h} R_{h} u-\Delta_{h} u_{h}\right\|_{L_{\infty}(\Omega)} \leq C h^{-2}\left(\left\|R_{h} u-u\right\|_{L_{\infty}(\Omega)}+\left\|u-u_{h}\right\|_{L_{\infty}(\Omega)}\right) .
$$

By (2.4), the estimate $\left\|R_{h} u-u\right\|_{L_{\infty}(\Omega)} \leq C h^{2}|\log h|^{2}\|\Delta u\|_{L_{\infty}(\Omega)}$ (cf. Lemma 4.1 in 12 ), and (2.5), we finally obtain

$$
\left\|R_{h} u-u\right\|_{L_{\infty}(\Omega)}+\left\|u-u_{h}\right\|_{L_{\infty}(\Omega)} \leq \frac{C h^{2}|\log h|^{2} \log \left(\frac{1}{\varepsilon}\right)}{\varepsilon}\|f\|_{W_{\infty}^{1}(\Omega)} .
$$

Combining estimates (2.9), (2.10), (2.11), (2.12), (2.13), and (2.14) we have the corollary.

In the next sections we will collect some results which we will use later.

\section{Global energy estimates}

For $v \in H^{1}(\Omega)$, define $P_{h}^{\varepsilon} v \in S_{h}^{r}$ by

$$
A_{\varepsilon}\left(v-P_{h}^{\varepsilon} v, \chi\right)=0, \text { for any } \chi \in S_{h}^{r},
$$

where

$$
A_{\varepsilon}(w, \chi):=\varepsilon^{2}(\nabla w, \nabla \chi)+(w, \chi) .
$$

Lemma 3.1. There exists a constant $C$ independent of $0<\varepsilon \leq 1$ and $0<h<1 / 2$ such that

$$
\left\|\nabla\left(v-P_{h}^{\varepsilon} v\right)\right\|_{L_{2}(\Omega)} \leq\left\{\begin{array}{l}
C\|v\|_{H^{1}(\Omega)}, \\
C h\|v\|_{H^{2}(\Omega)}
\end{array}\right.
$$

and

$$
\left\|v-P_{h}^{\varepsilon} v\right\|_{L_{2}(\Omega)} \leq\left\{\begin{array}{l}
C h\|v\|_{H^{1}(\Omega)}, \\
C h^{2}\|v\|_{H^{2}(\Omega)} .
\end{array}\right.
$$

The proof of this result, which is valid for $N \geq 2$, is in [11, Lemma 4.1.

\section{LOCAL ENERGY ESTimates}

In the results below we assume that $d \geq \underline{k} h$ for some positive constant $\underline{k}$.

Lemma 4.1. Let $0<\varepsilon \leq 1$ and $0<h \leq 1 / 2$ be parameters, and $v_{h} \in S_{h}^{r}\left(D_{d}\right)$ satisfy

$$
A_{\varepsilon}\left(v_{h}, \chi\right)=0, \text { for any } \chi \in S_{h}^{r}\left(D_{d}\right) .
$$

There exist positive constants $c_{1}$ and $C$ independent of $\varepsilon$ and $h$, such that

$$
\left\|v_{h}\right\|_{D}+d\left\|\nabla v_{h}\right\|_{D} \leq C e^{-\frac{c_{1} d}{\varepsilon+h}}\left\|v_{h}\right\|_{D_{d}} .
$$


Lemma 4.2. Let $0<\varepsilon \leq 1$ and $0<h \leq 1 / 2$ be parameters, and $v_{h} \in S_{h}^{r}\left(D_{d}\right)$ satisfy

$$
A_{\varepsilon}\left(v_{h}, \chi\right)=0, \text { for any } \chi \in S_{h}^{r}\left(D_{d}\right)
$$

There exist positive constants $c_{1}$ and $C$ independent of $\varepsilon$ and $h$, such that

$$
\left\|v-v_{h}\right\|_{H^{1}(D)} \leq C\left(\|\nabla(v-\chi)\|_{D_{d}}+d^{-1}\|v-\chi\|_{D_{d}}\right)+C d^{-1} e^{-\frac{c_{1} d}{\varepsilon+h}}\left\|v-v_{h}\right\|_{D_{d}} .
$$

The proofs of these two results are in 11, Lemma 5.1. and Lemma 5.2. respectively. Although the main result in that paper was done in the plane domains, the proofs of these lemmas are valid in any number of dimensions.

Lemma 4.3. Let $0<\varepsilon \leq 1$ and $0<h \leq 1 / 2$ be parameters, and $v_{h} \in S_{h}^{r}\left(D_{d}\right)$ satisfy

$$
A_{\varepsilon}\left(v-v_{h}, \chi\right)=0, \text { for any } \chi \in S_{h}^{r}\left(D_{d}\right) .
$$

There exist positive constants $c_{1}$ and $C$ independent of $\varepsilon$ and $h$, such that

$$
\left\|v-v_{h}\right\|_{D} \leq C h\left(\|\nabla(v-\chi)\|_{D_{2 d}}+d^{-1}\|v-\chi\|_{D_{2 d}}\right)+C e^{-\frac{c_{1} d}{\varepsilon+h}}\left\|v-v_{h}\right\|_{D_{2 d}} .
$$

Proof. Let $\omega \in C_{0}^{\infty}\left(D_{d}\right)$ be a cut-off function with the following properties:

$$
\omega \equiv 1 \text { on } D_{d} \text { and }\|\omega\|_{l, D_{2 d}} \leq C d^{-l}, l=0,1 \text {. }
$$

Define $\tilde{v}=\omega v$ and $\tilde{v}_{h}=P_{h}^{\varepsilon} \tilde{v}$. Then we have

$$
\left\|v-v_{h}\right\|_{D} \leq\left\|\tilde{v}-\tilde{v}_{h}\right\|_{D}+\left\|\tilde{v}_{h}-v_{h}\right\|_{D}
$$

Since $A^{\varepsilon}\left(\tilde{v}_{h}-v_{h}, \chi\right)=0$, for $\chi \in S_{h}^{r}\left(D_{d}\right)$, by Lemma 4.1 we have

$$
\begin{aligned}
\left\|\tilde{v}_{h}-v_{h}\right\|_{D} & \leq C e^{-\frac{c_{1} d}{\varepsilon+h}}\left\|\tilde{v}_{h}-v_{h}\right\|_{D_{d}} \\
& \leq C e^{-\frac{c_{1} d}{\varepsilon+h}}\left(\left\|\tilde{v}-\tilde{v}_{h}\right\|_{D_{d}}+\left\|v-v_{h}\right\|_{D_{d}}\right) .
\end{aligned}
$$

Thus we only need to estimate $\left\|\tilde{v}-\tilde{v}_{h}\right\|$. Using global energy estimates Lemma 3.1

$$
\left\|\tilde{v}-\tilde{v}_{h}\right\| \leq C h\|\tilde{v}\|_{1} \leq C h\left(\|\nabla v\|_{D_{2 d}}+d^{-1}\|v\|_{D_{2 d}}\right) .
$$

Combining estimates (4.1), (4.2), (4.3), and writing $v-v_{h}=(v-\chi)-\left(v_{h}-\chi\right)$ for $\chi \in S_{h}^{r}$, we complete the proof.

\section{Proof of the main Result: Part 1}

Let $x \in \bar{\tau}_{0}$. For any $\chi \in S_{h}^{r}$ using the triangle inequality and assumptions 2.2 and 2.3 we have

$$
\begin{aligned}
\left|\left(u-u_{h}\right)(x)\right| & \leq|(u-\chi)(x)|+C h^{-N / 2}\left\|\chi-u_{h}\right\|_{L_{2}\left(\tau_{0}\right)} \\
& \leq|(u-\chi)(x)|+C h^{-N / 2}\left(\|u-\chi\|_{L_{2}\left(\tau_{0}\right)}+\left\|u-u_{h}\right\|_{L_{2}\left(\tau_{0}\right)}\right) \\
& \leq C\|u-\chi\|_{L_{\infty}\left(\tau_{0}\right)}+C h^{-N / 2}\left\|u-u_{h}\right\|_{L_{2}\left(\tau_{0}\right)} .
\end{aligned}
$$

Define a function

$$
\eta(y)= \begin{cases}h^{-N / 2}\left(u-u_{h}\right)(y) /\left\|u-u_{h}\right\|_{L_{2}\left(\tau_{0}\right)}, & \text { for } y \in \tau_{0}, \\ 0, & \text { otherwise. }\end{cases}
$$

It is easy to see that $\|\eta\|_{L_{2}(\Omega)} \leq C h^{-N / 2}$ and $\|\eta\|_{L_{1}(\Omega)} \leq C$.

Define a function $g^{\varepsilon}$ to satisfy

$$
A_{\varepsilon}\left(v, g^{\varepsilon}\right)=(\eta, v), \text { for } v \in W_{2}^{1}(\Omega),
$$


and define $g_{h}^{\varepsilon} \in S_{h}^{r}$ to be a unique solution of

$$
A_{\varepsilon}\left(\chi, g^{\varepsilon}-g_{h}^{\varepsilon}\right)=0, \text { for all } \chi \in S_{h}^{r} .
$$

First we will show the global a priori estimates.

Lemma 5.1. There exists a constant $C$ independent of $0<\varepsilon \leq 1$ such that

$$
\begin{aligned}
\left\|g^{\varepsilon}\right\|_{L_{2}(\Omega)} & \leq C\|\eta\|_{L_{2}(\Omega)}=C h^{-N / 2}, \\
\left\|g^{\varepsilon}\right\|_{H^{1}(\Omega)} & \leq C \varepsilon^{-1}\|\eta\|_{L_{2}(\Omega)}=C \varepsilon^{-1} h^{-N / 2} \\
\left\|g^{\varepsilon}\right\|_{H^{2}(\Omega)} & \leq C \varepsilon^{-2}\|\eta\|_{L_{2}(\Omega)}=C \varepsilon^{-2} h^{-N / 2} .
\end{aligned}
$$

Proof. From (5.3) we have

$$
\varepsilon^{2}\left\|\nabla g^{\varepsilon}\right\|_{L_{2}(\Omega)}^{2}+\left\|g^{\varepsilon}\right\|_{L_{2}(\Omega)}^{2}=A_{\varepsilon}\left(g^{\varepsilon}, g^{\varepsilon}\right)=\left(\eta, g^{\varepsilon}\right) \leq\|\eta\|_{L_{2}(\Omega)}\left\|g^{\varepsilon}\right\|_{L_{2}(\Omega)} .
$$

Thus $\left\|g^{\varepsilon}\right\|_{L_{2}(\Omega)} \leq\|\eta\|_{L_{2}(\Omega)}$ and $\left\|\nabla g^{\varepsilon}\right\|_{L_{2}(\Omega)} \leq \varepsilon^{-1}\|\eta\|_{L_{2}(\Omega)}$, which proves the first two estimates.

To prove the last estimate we notice that

$$
\left\|g^{\varepsilon}\right\|_{H^{2}(\Omega)} \leq C\left\|-\Delta g^{\varepsilon}+g^{\varepsilon}\right\|_{L_{2}(\Omega)},
$$

hence

$$
\begin{aligned}
\left\|g^{\varepsilon}\right\|_{H^{2}(\Omega)} & \leq C \varepsilon^{-2}\left\|-\varepsilon^{2} \Delta g^{\varepsilon}+g^{\varepsilon}\right\|_{L_{2}(\Omega)}+C\left(1+\varepsilon^{-2}\right)\left\|g^{\varepsilon}\right\|_{L_{2}(\Omega)} \\
& \leq C \varepsilon^{-2}\|\eta\|_{L_{2}(\Omega)}=C \varepsilon^{-2} h^{-N / 2},
\end{aligned}
$$

which completes the proof of the lemma.

Thus we have

$$
\begin{aligned}
& h^{-N / 2}\left\|u-u_{h}\right\|_{L_{2}\left(\tau_{0}\right)}=\left(u-u_{h}, \eta\right)=A_{\varepsilon}\left(u-u_{h}, g^{\varepsilon}\right)=A_{\varepsilon}\left(u-u_{h}, g^{\varepsilon}-g_{h}^{\varepsilon}\right) \\
& =A_{\varepsilon}\left(u-\chi, g^{\varepsilon}-g_{h}^{\varepsilon}\right) \\
& =-\varepsilon^{2} \sum_{i}\left(\int_{\tau_{i}^{h}}(u-\chi) \Delta\left(g^{\varepsilon}-g_{h}^{\varepsilon}\right)+\oint_{\partial \tau_{i}^{h}}(u-\chi) \nabla\left(g^{\varepsilon}-g_{h}^{\varepsilon}\right) \cdot n\right) \\
& +\left(u-\chi, g^{\varepsilon}-g_{h}^{\varepsilon}\right) .
\end{aligned}
$$

Letting $F^{\varepsilon} \equiv g^{\varepsilon}-g_{h}^{\varepsilon}$ and using Trace Inequality 2.1 we have,

$$
\begin{aligned}
h^{-N / 2}\left\|u-u_{h}\right\|_{L_{2}\left(\tau_{0}\right)} \leq & C\left\|e^{-c \frac{|x-y|}{\varepsilon+h}}(u-\chi)\right\|_{L_{\infty}(\Omega), \sigma, s}\left(\varepsilon^{2}\left\|e^{c \frac{|x-y|}{\varepsilon+h}} D^{2} F^{\varepsilon}\right\|_{L_{1}(\Omega), \sigma,-s}^{(h)}\right. \\
& \left.+\varepsilon^{2} h^{-1}\left\|e^{c \frac{|x-y|}{\varepsilon+h}} \nabla F^{\varepsilon}\right\|_{L_{1}(\Omega), \sigma,-s}+\left\|e^{c \frac{|x-y|}{\varepsilon+h}} F^{\varepsilon}\right\|_{L_{1}(\Omega), \sigma,-s}\right) .
\end{aligned}
$$

By the triangle inequality

$$
\begin{aligned}
\left\|e^{c \frac{|x-y|}{\varepsilon+h}} D^{2} F^{\varepsilon}\right\|_{L_{1}(\Omega), \sigma,-s}^{(h)} & \leq\left\|e^{c \frac{|x-y|}{\varepsilon+h}} D^{2}\left(g^{\varepsilon}-\chi\right)\right\|_{L_{1}(\Omega), \sigma,-s}^{(h)} \\
& +\left\|e^{c \frac{|x-y|}{\varepsilon+h}} D^{2}\left(g_{h}^{\varepsilon}-\chi\right)\right\|_{L_{1}(\Omega), \sigma,-s}^{(h)}, \text { for any } \chi \in S_{h}^{r} .
\end{aligned}
$$

Let $y_{\tau} \in \tau$ be the center of the circumscribed sphere over an element $\tau$. Using the triangle inequality $|x-y| \leq\left|x-y_{\tau}\right|+\left|y_{\tau}-y\right|$, assumption 2.1, and inverse 
inequality 2.2 in the case $D=\tau$, we have

$$
\begin{aligned}
& \left\|e^{c \frac{|x-y|}{\varepsilon+h}} D^{2}\left(g_{h}^{\varepsilon}-\chi\right)\right\|_{L_{1}(\Omega), \sigma,-s}^{(h)}=\sum_{\tau} \int_{\tau}\left|e^{c \frac{|x-y|}{\varepsilon+h}} D^{2}\left(g_{h}^{\varepsilon}-\chi\right)\left(\frac{h+|x-y|}{h}\right)^{s}\right| \\
& \leq \sum_{\tau} e^{c \frac{|x-y \tau|+\bar{k} h}{\varepsilon+h}}\left(\frac{h+\left|x-y_{\tau}\right|+\bar{k} h}{h}\right)^{s} \int_{\tau}\left|D^{2}\left(g_{h}^{\varepsilon}-\chi\right)\right| \\
& \leq C h^{-1} \sum_{\tau} e^{c \frac{\left|x-y_{\tau}\right|+\bar{k} h}{\varepsilon+h}}\left(\frac{h+\left|x-y_{\tau}\right|+\bar{k} h}{h}\right)^{s}\left(\int_{\tau}\left|\nabla\left(g^{\varepsilon}-\chi\right)\right|+\left|\nabla\left(g^{\varepsilon}-g_{h}^{\varepsilon}\right)\right|\right) .
\end{aligned}
$$

Using the triangle inequality $-|x-y| \leq\left|y_{\tau}-y\right|-\left|x-y_{\tau}\right|$, we have

$$
\begin{aligned}
& \sum_{\tau} e^{c \frac{\left|x-y_{\tau}\right|+\bar{k} h}{\varepsilon+h}}\left(\frac{h+\left|x-y_{\tau}\right|+\bar{k} h}{h}\right)^{s}\left(\int_{\tau}\left|\nabla\left(g^{\varepsilon}-\chi\right)\right|+\left|\nabla\left(g^{\varepsilon}-g_{h}^{\varepsilon}\right)\right|\right) \\
& \leq \sum_{\tau} e^{c \frac{2 \bar{k} h}{\varepsilon+h}}(1+2 \bar{k})^{s} \int_{\tau}\left|e^{c \frac{|x-y|}{\varepsilon+h}} \nabla\left(g^{\varepsilon}-\chi\right)\left(\frac{h+|x-y|}{h}\right)^{s}\right| \\
& +\sum_{\tau} e^{c \frac{2 \bar{k} h}{\varepsilon+h}}(1+2 \bar{k})^{s} \int_{\tau}\left|e^{c \frac{|x-y|}{\varepsilon+h}} \nabla\left(g^{\varepsilon}-g_{h}^{\varepsilon}\right)\left(\frac{h+|x-y|}{h}\right)^{s}\right| \\
& \leq e^{2 c \bar{k}}(1+2 \bar{k})^{s}\left(\left\|e^{c \frac{|x-y|}{\varepsilon+h}} \nabla\left(g^{\varepsilon}-\chi\right)\right\|_{L_{1}(\Omega), \sigma,-s}+\left\|e^{c \frac{|x-y|}{\varepsilon+h}} \nabla F^{\varepsilon}\right\|_{L_{1}(\Omega), \sigma,-s}\right) .
\end{aligned}
$$

Thus, we have shown

$$
\begin{aligned}
& \left\|e^{c \frac{|x-y|}{\varepsilon+h}} D^{2} F^{\varepsilon}\right\|_{L_{1}(\Omega), \sigma,-s}^{(h)} \leq C h^{-1}\left\|e^{c \frac{|x-y|}{\varepsilon+h}} \nabla F^{\varepsilon}\right\|_{L_{1}(\Omega), \sigma,-s} \\
& +C h^{-1}\left\|e^{c \frac{|x-y|}{\varepsilon+h}} \nabla\left(g^{\varepsilon}-\chi\right)\right\|_{L_{1}(\Omega), \sigma,-s}+\left\|e^{c \frac{|x-y|}{\varepsilon+h}} D^{2}\left(g^{\varepsilon}-\chi\right)\right\|_{L_{1}(\Omega), \sigma,-s}^{(h)} .
\end{aligned}
$$

Putting it all together, we have

$$
\begin{aligned}
\mid(u- & \left.u_{h}\right)(x) \mid \leq C\left\|e^{-c \frac{|x-y|}{\varepsilon+h}}(u-\chi)\right\|_{L_{\infty}(\Omega), \sigma, s} \\
& \times\left(1+\varepsilon^{2} h^{-1}\left\|e^{c \frac{|x-y|}{\varepsilon+h}} \nabla F^{\varepsilon}\right\|_{L_{1}(\Omega), \sigma,-s}+\left\|e^{c \frac{|x-y|}{\varepsilon+h}} F^{\varepsilon}\right\|_{L_{1}(\Omega), \sigma,-s}\right. \\
& \left.+\varepsilon^{2} h^{-1}\left\|e^{c \frac{|x-y|}{\varepsilon+h}} \nabla\left(g^{\varepsilon}-\chi\right)\right\|_{L_{1}(\Omega), \sigma,-s}+\varepsilon^{2}\left\|e^{c \frac{|x-y|}{\varepsilon+h}} D^{2}\left(g^{\varepsilon}-\chi\right)\right\|_{L_{1}(\Omega), \sigma,-s}^{(h)}\right) .
\end{aligned}
$$

Thus in order to prove the theorem we need to show that

$$
\begin{aligned}
I_{1}=\varepsilon^{2} h^{-1}\left\|e^{c \frac{|x-y|}{\varepsilon+h}} \nabla\left(g^{\varepsilon}-\chi\right)\right\|_{L_{1}(\Omega), \sigma,-s} \\
\quad+\varepsilon^{2}\left\|e^{c \frac{|x-y|}{\varepsilon+h}} D^{2}\left(g^{\varepsilon}-\chi\right)\right\|_{L_{1}(\Omega), \sigma,-s}^{(h)} \leq C \ell_{h}
\end{aligned}
$$

and

$$
I_{2}=\varepsilon^{2} h^{-1}\left\|e^{c \frac{|x-y|}{\varepsilon+h}} \nabla F^{\varepsilon}\right\|_{L_{1}(\Omega), \sigma,-s}+\left\|e^{c \frac{|x-y|}{\varepsilon+h}} F^{\varepsilon}\right\|_{L_{1}(\Omega), \sigma,-s} \leq C \ell_{h} .
$$

\section{Proof of the main Results: Part 2}

To prove (5.9), we shall decompose $\Omega$ into "annuli". For $j$ an integer, let $d_{j}=$ $2^{-j}$ and $\Omega_{j}=\left\{y \in \Omega: d_{j} \leq|y-x| \leq 2 d_{j}\right\}$. Then, with $J_{0}$ fixed such that $|y-x| \leq 2 d_{J_{0}}=2^{1-J_{0}}$ in $\Omega$, and any $J_{*}>J_{0}$

$$
\Omega=\left(\bigcup_{j=J_{0}}^{J_{*}} \Omega_{j}\right) \cup \Omega_{*}, \text { where } \Omega_{*}=\left\{y \in \Omega:|y-x| \leq d_{J_{*}}\right\} .
$$


We shall refer to $\Omega_{*}$ as the "innermost" set. Ultimately, we shall choose $J_{*}=J_{*}(h)$ such that $d_{J_{*}} \approx C_{*} h$ for small $h$, where $C_{*}$ is a sufficiently large number to be chosen later. Note that then $J_{*} \approx C|\log h|$. Constants $C$ and $c$ will, as usual, change freely but will be independent of $C_{*}$. We shall write $\sum_{*, j}$ when the innermost set is included and $\sum_{j}$ when it is not. We also define $\Omega_{j}^{\prime}=\Omega_{j-1} \cup \Omega_{j} \cup \Omega_{j+1}, \Omega_{j}^{\prime \prime}=\left(\Omega_{j}^{\prime}\right)^{\prime}$, and so on.

Proposition 6.1. There exist constants $C$ and $c$ such that $I_{1} \leq C C_{*}^{N / 2+s} e^{c \frac{C_{*} h}{\varepsilon+h}}+$ $C \ell_{h}$, where $I_{1}$ is defined in (5.9).

Proof. First we shall bound the second term in $I_{1}$ on $\Omega_{*}$. Since on $\Omega_{*}$ the weights $e^{\frac{c|y-x|}{\varepsilon+h}} \leq e^{\frac{C_{*} h}{\varepsilon+h}}$ and $\sigma \leq C_{*}$, it is sufficient to estimate $\left\|g^{\varepsilon}-I_{h} g^{\varepsilon}\right\|_{W_{1}^{2}\left(\Omega_{*}\right)}$. Using the Cauchy-Schwarz inequality, the local approximation assumption 2.3. a priori estimate of Lemma 5.1 and (5.2), we have

$\left\|g^{\varepsilon}-I_{h} g^{\varepsilon}\right\|_{W_{1}^{2}\left(\Omega_{*}\right)} \leq C\left(C_{*} h\right)^{N / 2}\left\|g^{\varepsilon}\right\|_{W_{2}^{2}(\Omega)} \leq C\left(C_{*} h\right)^{N / 2} \varepsilon^{-2}\|\eta\|_{L_{2}(\Omega)} \leq C C_{*}^{N / 2} \varepsilon^{-2}$.

To estimate $I_{1}$ on $\Omega \backslash \Omega_{*}^{\prime}$ we use the representation $g^{\varepsilon}(x)=\int_{\Omega} K^{\varepsilon}(x, y) \eta(y) d y$. The Green's function $K^{\varepsilon}(x, y)$ is singular only for $x=y$. Hence if $x \notin \operatorname{supp}(\eta)$, the representation $D^{\alpha} g^{\varepsilon}(x)=\int_{\Omega} D_{x}^{\alpha} K^{\varepsilon}(x, y) \eta(y) d y$ is valid for multi-index $\alpha$.

Using local approximation 2.3 and Lemma 2.2. for any $|\alpha|=r$ and $c<c_{0}$ we have

$$
\begin{aligned}
& \left\|e^{c \frac{|y-x|}{\varepsilon+h}} D^{2}\left(g^{\varepsilon}-I_{h} g^{\varepsilon}\right)\right\|_{L^{1}\left(\Omega \backslash \Omega_{*}^{\prime}\right), \sigma,-s}^{(h)} \leq \sum_{j=J_{0}}^{J_{*}-1}\left(d_{j} / h\right)^{s} e^{\frac{c d_{j}}{h+\varepsilon}}\left\|g^{\varepsilon}-I_{h} g^{\varepsilon}\right\|_{W_{1}^{2}\left(\Omega_{j}\right)}^{(h)} \\
& \leq C \sum_{j=J_{0}}^{J_{*}-1}\left(d_{j} / h\right)^{s} e^{\frac{c d_{j}}{h+\varepsilon}} h^{r-2}\left\|g^{\varepsilon}\right\|_{W_{1}^{r}\left(\Omega_{j}\right)} \\
& \leq C \varepsilon^{-2} \sum_{j=J_{0}}^{J_{*}-1}\left(d_{j} / h\right)^{s} e^{\frac{c d_{j}}{h+\varepsilon}} h^{r-2} d_{j}^{N} e^{-c_{0} \frac{d_{j}}{\varepsilon}} d_{j}^{2-N-r}\|\eta\|_{L_{1}(\Omega)} \\
& \leq C \varepsilon^{-2} \sum_{j=J_{0}}^{J_{*}-1}\left(\frac{h}{d_{j}}\right)^{r-2-s} e^{-\tilde{c} \frac{d_{j}}{\varepsilon}} \leq \begin{cases}C \varepsilon^{-2}, & \text { if } r-2>s \text { or } \varepsilon=O(h), \\
C \varepsilon^{-2}|\log h|, & \text { if } r-2=s \text { and } \varepsilon \gg h .\end{cases}
\end{aligned}
$$

The proof is very similar for the other term in $I_{1}$.

To conclude the proof of Theorem 2.1, it remains to prove the following result.

Proposition 6.2. There exist constants $c, C$, and $C_{*}$, with the latter large enough, such that $I_{2} \leq C C_{*}^{N / 2+s} e^{c \frac{C_{*} h}{h+\varepsilon}}+C \ell_{h}$, where $I_{2}$ is defined in (5.9).

Proof. In this proof, almost all norms occurring in the estimates will be $L_{2}$ based. We shall write $\|v\|_{D}$ for $L_{2}$-norms over a set $D$ and $\|v\|_{k, D}$ when up to $k$ spatial derivatives are included.

Using Cauchy-Schwarz inequality

$$
I_{2} \leq \sum_{*, j}\left(d_{j} / h\right)^{s}\left(2 d_{j}\right)^{N / 2} e^{\frac{c d_{j}}{h+\varepsilon}}\left(\varepsilon^{2} h^{-1}\left\|\nabla F^{\varepsilon}\right\|_{\Omega_{j}}+\left\|F^{\varepsilon}\right\|_{\Omega_{j}}\right) .
$$

The part of $I_{2}$ over $\Omega_{*}$, which we will call $I_{2}^{*}$, can be bounded by

$$
\begin{aligned}
I_{2}^{*} & \leq C C_{*}^{N / 2+s} h^{N / 2} e^{c \frac{C_{*} h}{h+\varepsilon}}\left(\left\|F^{\varepsilon}\right\|_{\Omega}+\varepsilon^{2} h^{-1}\left\|\nabla F^{\varepsilon}\right\|_{\Omega}\right) \\
& \leq C C_{*}^{N / 2+s} h^{N / 2} e^{c \frac{C_{*} h}{h+\varepsilon}}\left(\left\|g^{\varepsilon}\right\|_{\Omega}+\left\|g_{h}^{\varepsilon}\right\|_{\Omega}+\varepsilon^{2}\left\|g^{\varepsilon}\right\|_{2, \Omega}\right)
\end{aligned}
$$


by using the global estimate from Lemma 3.1. Using a priori estimates in Lemma 5.1 and the fact that $\left\|g_{h}^{\varepsilon}\right\|_{\Omega} \leq\|\eta\|_{\Omega}$, we get

$$
I_{2}^{*} \leq C C_{*}^{N / 2+s} h^{N / 2} e^{c \frac{C_{*} h}{h+\varepsilon}}\|\eta\|_{\Omega} \leq C C_{*}^{N / 2+s} e^{c \frac{C_{*} h}{h+\varepsilon}} .
$$

The remaining terms are bounded by $C d_{j}^{N / 2}\left(d_{j} / h\right)^{s} e^{\frac{c d_{j}}{h+\varepsilon}} M_{j}$, where

$$
M_{j}=\left\|F^{\varepsilon}\right\|_{\Omega_{j}}+\varepsilon^{2} h^{-1}\left\|\nabla F^{\varepsilon}\right\|_{\Omega_{j}} .
$$

Thus so far we have

$$
I_{2} \leq C C_{*}^{N / 2+s} e^{c \frac{C_{*} h}{h+\varepsilon}}+C M, \text { where } M=\sum_{j} d_{j}^{N / 2}\left(d_{j} / h\right)^{s} e^{\frac{c d_{j}}{h+\varepsilon}} M_{j} .
$$

To treat the terms involved in $M_{j}$, we shall consider two cases, $\varepsilon \leq h$ and $\varepsilon>h$.

6.1. Case 1: $\varepsilon \leq h$.

$$
M \leq \sum_{j} d_{j}^{N / 2}\left(d_{j} / h\right)^{s} e^{\frac{c d_{j}}{\varepsilon+h}}\left(\left\|g^{\varepsilon}\right\|_{\Omega_{j}}+\left\|g_{h}^{\varepsilon}\right\|_{\Omega_{j}}+\varepsilon^{2} h^{-1}\left\|\nabla g^{\varepsilon}\right\|_{\Omega_{j}}+\varepsilon^{2} h^{-1}\left\|\nabla g_{h}^{\varepsilon}\right\|_{\Omega_{j}}\right) .
$$

Using the Green's function representation and Lemma 2.2 for $N \geq 3$, we have

$$
\left|g^{\varepsilon}(x)\right| \leq \int_{\Omega}\left|K^{\varepsilon}(x, y)\right| \cdot|\eta(y)| d y \leq C \varepsilon^{-2} d_{j}^{2-N} e^{-c_{0} \frac{d_{j}}{\varepsilon}}\|\eta\|_{L_{1}(\Omega)} .
$$

Hence,

$$
\left\|g^{\varepsilon}\right\|_{\Omega_{j}} \leq C d_{j}^{N / 2} \varepsilon^{-2} d_{j}^{2-N} e^{-c_{0} \frac{d_{j}}{\varepsilon}}\|\eta\|_{L_{1}} \leq C d_{j}^{2-N / 2} \varepsilon^{-2} e^{-c_{0} \frac{d_{j}}{\varepsilon}} .
$$

Using the fact that $\varepsilon \leq h$,

$$
\sum_{j} d_{j}^{N / 2}\left(d_{j} / h\right)^{s} e^{\frac{c d_{j}}{\varepsilon+h}}\left\|g^{\varepsilon}\right\|_{\Omega_{j}} \leq C \sum_{j} e^{-\tilde{c} \frac{d_{j}}{\varepsilon}}\left(d_{j} / \varepsilon\right)^{2+s} \leq C .
$$

Very similarly

$$
\left\|\nabla g^{\varepsilon}\right\|_{\Omega_{j}} \leq C d_{j}^{1-N / 2} \varepsilon^{-2} e^{-c_{0} \frac{d_{j}}{\varepsilon}},
$$

and using the fact that $\varepsilon \leq h$,

$$
\sum_{j} d_{j}^{N / 2}\left(d_{j} / h\right)^{s} e^{\frac{c d_{j}}{\varepsilon+h}} \varepsilon^{2} h^{-1}\left\|\nabla g^{\varepsilon}\right\|_{\Omega_{j}} \leq C \sum_{j} e^{-\tilde{c} \frac{d_{j}}{\varepsilon}}\left(d_{j} / \varepsilon\right)^{1+s} \leq C .
$$

The case $N=2$ is similar, and we leave it to the reader.

Applying Lemma 4.1 to $\left\|g_{h}^{\varepsilon}\right\|_{\Omega_{j}}$ and $\left\|\nabla g_{h}^{\varepsilon}\right\|_{\Omega_{j}}$, we get

$$
\left\|g_{h}^{\varepsilon}\right\|_{\Omega_{j}}+d_{j}\left\|\nabla g_{h}^{\varepsilon}\right\|_{\Omega_{j}} \leq C e^{-c_{1} \frac{d_{j}}{h}}\left\|g_{h}^{\varepsilon}\right\|_{\Omega_{j}^{\prime}} \leq C e^{-c_{1} \frac{d_{j}}{h}}\|\eta\|_{\Omega} \leq C e^{-c_{1} \frac{d_{j}}{h}} h^{-N / 2} .
$$

Thus again using the fact that $\varepsilon \leq h$,

$$
\begin{aligned}
& \sum_{j} d_{j}^{N / 2}\left(d_{j} / h\right)^{s} e^{\frac{c d_{j}}{h}}\left(\left\|g_{h}^{\varepsilon}\right\|_{\Omega_{j}}+\varepsilon^{2} h^{-1}\left\|\nabla g_{h}^{\varepsilon}\right\|_{\Omega_{j}}\right) \\
& \leq C \sum_{j} e^{-\tilde{c} \frac{d_{j}}{h}}\left(d_{j} / h\right)^{N / 2+s}+C \sum_{j} e^{-\tilde{c} \frac{d_{j}}{h}}\left(d_{j} / h\right)^{N / 2+1+s} \leq C .
\end{aligned}
$$

Combining estimates (6.5), (6.6), and (6.7) we complete the proof in the case when $\varepsilon \leq h$. 
6.2. Case 2: $\varepsilon>h$. To treat the terms involved in $M_{j}$ in (6.4), we shall use the local energy-based estimates from Section 4 .

By Lemma 4.2 we have

$$
\left\|\nabla F^{\varepsilon}\right\|_{\Omega_{j}} \leq C\left(\left\|\nabla\left(g^{\varepsilon}-\chi\right)\right\|_{\Omega_{j}^{\prime}}+d_{j}^{-1}\left\|g^{\varepsilon}-\chi\right\|_{\Omega_{j}^{\prime}}\right)+C d_{j}^{-1} e^{-\frac{c_{1} d_{j}}{\varepsilon+h}}\left\|F^{\varepsilon}\right\|_{\Omega_{j}^{\prime}},
$$

for any $\chi \in S_{h}^{r}$. Taking $\chi=I_{h} g^{\varepsilon}$, using Green's function representation and the fact that $h<d_{j}$, we can estimate the first two terms in (6.8) as

$$
\begin{aligned}
& \left\|\nabla\left(g^{\varepsilon}-I_{h} g^{\varepsilon}\right)\right\|_{\Omega_{j}^{\prime}}+d_{j}^{-1}\left\|g^{\varepsilon}-I_{h} g^{\varepsilon}\right\|_{\Omega_{j}^{\prime}} \\
& \quad \leq C h^{r-1}\left\|g^{\varepsilon}\right\|_{r, \Omega_{j}^{\prime \prime}} \leq C h^{r-1} \varepsilon^{-2} e^{-c_{0} \frac{d_{j}}{\varepsilon}} d_{j}^{2-N / 2-r} .
\end{aligned}
$$

Hence the contribution to $M$ is bounded by

$$
\begin{gathered}
C \sum_{j} d_{j}^{N / 2}\left(d_{j} / h\right)^{s} e^{\frac{c d_{j}}{h+\varepsilon}} h^{r-2} e^{-c_{0} \frac{d_{j}}{\varepsilon}} d_{j}^{2-N / 2-r} \\
\leq C \sum_{j}\left(h / d_{j}\right)^{r-2-s} e^{-\tilde{c} \frac{d_{j}}{\varepsilon}} \leq C \ell_{h} .
\end{gathered}
$$

We now apply Lemma 4.3 to the other term in $M_{j}$, namely $\left\|F^{\varepsilon}\right\|_{\Omega_{j}}$ :

$$
\left\|F^{\varepsilon}\right\|_{\Omega_{j}} \leq C h\left(\left\|\nabla\left(g^{\varepsilon}-\chi\right)\right\|_{\Omega_{j}^{\prime}}+d_{j}^{-1}\left\|g^{\varepsilon}-\chi\right\|_{\Omega_{j}^{\prime}}\right)+C e^{-\frac{c_{1} d_{j}}{\varepsilon+h}}\left\|F^{\varepsilon}\right\|_{\Omega_{j}^{\prime}} .
$$

Using estimates (6.9) and the fact that $\varepsilon>h$, we see that the contribution to $M$ is bounded by

$$
\begin{gathered}
C \sum_{j} d_{j}^{N / 2}\left(d_{j} / h\right)^{s} e^{\frac{c d_{j}}{h+\varepsilon}} h^{r} \varepsilon^{-2} e^{-c_{0} \frac{d_{j}}{\varepsilon}} d_{j}^{2-N / 2-r} \\
\leq C \sum_{j}\left(h / d_{j}\right)^{r-2-s} e^{-\tilde{c} \frac{d_{j}}{\varepsilon}} \leq C \ell_{h}
\end{gathered}
$$

Thus we have

$$
M \leq C \ell_{h}+C \sum_{j} d_{j}^{N / 2}\left(d_{j} / h\right)^{s}\left(1+\varepsilon^{2} h^{-1} d_{j}^{-1}\right) e^{-\tilde{c} \frac{d_{j}}{\varepsilon}}\left\|F^{\varepsilon}\right\|_{\Omega_{j}^{\prime}} .
$$

In the following lemma we will estimate $\left\|F^{\varepsilon}\right\|_{\Omega_{j}^{\prime}}$ by a duality argument.

Lemma 6.3. The following estimate holds:

$$
\begin{aligned}
\left\|F^{\varepsilon}\right\|_{\Omega_{j}^{\prime}} & \leq C h\left\|\nabla F^{\varepsilon}\right\|_{\Omega_{j}^{\prime \prime \prime}}+C h^{2} \varepsilon^{-2}\left\|F^{\varepsilon}\right\|_{\Omega_{j}^{\prime \prime \prime}} \\
& +C h^{r} e^{-c_{0} \frac{d_{j}}{\varepsilon}} d_{j}^{2-N / 2-r} \varepsilon^{-2}\left(\varepsilon^{2} h^{-1}\left\|\nabla F^{\varepsilon}\right\|_{L_{1}(\Omega)}+\left\|F^{\varepsilon}\right\|_{L_{1}(\Omega)}\right) .
\end{aligned}
$$

Proof. Using $(v, w)_{D}$ for the $L_{2}$ inner product over a set $D$, we have

$$
\left\|F^{\varepsilon}\right\|_{\Omega_{j}^{\prime}}=\sup \left\{\left(F^{\varepsilon}, v\right)_{\Omega}: \operatorname{supp} v \subset \Omega_{j}^{\prime},\|v\|_{\Omega_{j}^{\prime}}=1\right\} .
$$


For each such fixed $v$, let $w$ solve the dual problem $-\varepsilon^{2} \Delta w+w=v$ in $\Omega$. Integrating by parts, we obtain for any $\chi \in S_{h}^{r}$,

$$
\begin{aligned}
\left(F^{\varepsilon}, v\right)_{\Omega}= & \varepsilon^{2}\left(\nabla F^{\varepsilon}, \nabla w\right)_{\Omega}+\left(F^{\varepsilon}, w\right)_{\Omega} \\
= & \varepsilon^{2}\left(\nabla F^{\varepsilon}, \nabla(w-\chi)\right)_{\Omega}+\left(F^{\varepsilon}, w-\chi\right)_{\Omega} \\
= & \varepsilon^{2}\left(\nabla F^{\varepsilon}, \nabla(w-\chi)\right)_{\Omega_{j}^{\prime \prime \prime}}+\left(F^{\varepsilon}, w-\chi\right)_{\Omega_{j}^{\prime \prime \prime}} \\
& +\varepsilon^{2}\left(\nabla F^{\varepsilon}, \nabla(w-\chi)\right)_{\Omega \backslash \Omega_{j}^{\prime \prime \prime}}+\left(F^{\varepsilon}, w-\chi\right)_{\Omega \backslash \Omega_{j}^{\prime \prime \prime}} \\
\leq & \varepsilon^{2}\left\|\nabla F^{\varepsilon}\right\|_{\Omega_{j}^{\prime \prime \prime}}\|\nabla(w-\chi)\|_{\Omega}+\left\|F^{\varepsilon}\right\|_{\Omega_{j}^{\prime \prime \prime}}\|w-\chi\|_{\Omega} \\
& +\varepsilon^{2}\left\|\nabla F^{\varepsilon}\right\|_{L_{1}(\Omega)}\|\nabla(w-\chi)\|_{L_{\infty}\left(\Omega \backslash \Omega_{j}^{\prime \prime \prime}\right)} \\
& +\left\|F^{\varepsilon}\right\|_{L_{1}(\Omega)}\|w-\chi\|_{L_{\infty}\left(\Omega \backslash \Omega_{j}^{\prime \prime \prime}\right)} .
\end{aligned}
$$

Take $\chi=I_{h} w$. Using the approximation and the global stability, we obtain

$$
\|w-\chi\|_{\Omega}+h\|\nabla(w-\chi)\|_{\Omega} \leq C h^{2}\|w\|_{H^{2}(\Omega)} \leq C \frac{h^{2}}{\varepsilon^{2}}\|v\|_{\Omega}=C \frac{h^{2}}{\varepsilon^{2}},
$$

and

$$
\begin{aligned}
& \|w-\chi\|_{L_{\infty}\left(\Omega \backslash \Omega_{j}^{\prime \prime \prime}\right)}+h\|\nabla(w-\chi)\|_{L_{\infty}\left(\Omega \backslash \Omega_{j}^{\prime \prime \prime}\right)} \leq C h^{r}\|w\|_{W_{\infty}^{r}\left(\Omega \backslash \Omega_{j}^{\prime \prime}\right)} \\
& \quad \leq C \frac{h^{r}}{\varepsilon^{2}} e^{-c_{0} \frac{d_{j}}{\varepsilon}} d_{j}^{2-N / 2-r} .
\end{aligned}
$$

In the last estimate we used the Green's function representation, Lemma 2.2. and Cauchy-Schwarz inequality, i.e.

$$
\begin{aligned}
\left|D^{r} w(x)\right| \leq C \int_{\Omega_{j}^{\prime}}\left|D_{x}^{r} K(x, y) v(y)\right| d y & \leq C \varepsilon^{-2} e^{-c_{0} \frac{d_{j}}{\varepsilon}} d_{j}^{2-N-r}\|v\|_{L_{1}\left(\Omega_{j}^{\prime}\right)} \\
& \leq C \varepsilon^{-2} e^{-c_{0} \frac{d_{j}}{\varepsilon}} d_{j}^{2-N / 2-r} .
\end{aligned}
$$

Combining estimates (6.15), (6.16), (6.17), and taking the supremum over $v$, we have the lemma.

Now we are ready to conclude the proof of Proposition 6.2 By the lemma above and (6.13), we have

$$
\begin{aligned}
M & \leq C \ell_{h}+C \sum_{j} d_{j}^{N / 2}\left(d_{j} / h\right)^{s}\left(\varepsilon^{-2} h^{2}+h d_{j}^{-1}\right)\left(\varepsilon^{2} h^{-1}\left\|\nabla F^{\varepsilon}\right\|_{\Omega_{j}^{\prime \prime \prime}}+\left\|F^{\varepsilon}\right\|_{\Omega_{j}^{\prime \prime \prime}}\right) \\
& +C\left(\varepsilon^{2} h^{-1}\left\|\nabla F^{\varepsilon}\right\|_{L_{1}(\Omega)}+\left\|F^{\varepsilon}\right\|_{L_{1}(\Omega)}\right) \sum_{j}\left(d_{j} / h\right)^{s}\left(\varepsilon^{-2} h^{2}+h d_{j}^{-1}\right) h^{r-2} e^{-c \frac{d_{j}}{\varepsilon}} d_{j}^{2-r} .
\end{aligned}
$$

In the first sum on the right hand side we can replace $\varepsilon^{2} h^{-1}\left\|\nabla F^{\varepsilon}\right\|_{\Omega_{j}^{\prime \prime \prime}}+\left\|F^{\varepsilon}\right\|_{\Omega_{j}^{\prime \prime \prime}}$ by $\varepsilon^{2} h^{-1}\left\|\nabla F^{\varepsilon}\right\|_{\Omega_{j}}+\left\|F^{\varepsilon}\right\|_{\Omega_{j}}$. This multiplies the sum at most by seven. The overshooting contribution near the innermost $\Omega_{*}$ is estimated as before by $C C_{*}^{N / 2+s} e^{c \frac{C_{*} h}{h+\varepsilon}}$. 
Using that $\sigma^{-s} \geq 1$ and $e^{c \frac{|x-y|}{\varepsilon+h}} \geq 1$, and the inequality $e^{-c \frac{d_{j}}{\varepsilon}} \leq C\left(\frac{\varepsilon}{d_{j}}\right)^{p}$ for any $p>0$, from (6.18) we obtain

$$
\begin{aligned}
M & \leq C C_{*}^{N / 2+s} e^{c \frac{C_{*} h}{h+\varepsilon}} \\
& +C \ell_{h}+C \sum_{j} d_{j}^{N / 2}\left(d_{j} / h\right)^{s} h d_{j}^{-1} e^{\frac{c d_{j}}{\varepsilon+h}}\left(\varepsilon^{2} h^{-1}\left\|\nabla F^{\varepsilon}\right\|_{\Omega_{j}}+\left\|F^{\varepsilon}\right\|_{\Omega_{j}}\right) \\
& +C\left(\left\|e^{c \frac{|x-y|}{\varepsilon+h}} \nabla F^{\varepsilon}\right\|_{L_{1}(\Omega), \sigma,-s}+\varepsilon^{2} h^{-1}\left\|e^{c \frac{|x-y|}{\varepsilon+h}} F^{\varepsilon}\right\|_{L_{1}(\Omega), \sigma,-s}\right) \sum_{j}\left(h / d_{j}\right)^{r-1-s} .
\end{aligned}
$$

Recalling the definitions of $I_{2}, M_{j}$, and $M$, (5.9), (6.3), and (6.4) respectively, and using that $h / d_{j} \leq C_{*}^{-1}$, we have

$$
M \leq C C_{*}^{N / 2+s} e^{c \frac{C_{*} h}{h+\varepsilon}}+C \ell_{h}+C C_{*}^{-1} M+I_{2} C \sum_{j}\left(h / d_{j}\right)^{r-1-s} .
$$

By choosing $C_{*}$ large enough, from (6.19) we can conclude that

$$
M \leq C C_{*}^{N / 2+s} e^{c \frac{C_{*} h}{h+\varepsilon}}+C \ell_{h}+I_{2} C \sum_{j}\left(h / d_{j}\right)^{r-1-s} .
$$

Inserting it into (6.4), we have

$$
I_{2} \leq C C_{*}^{N / 2+s} e^{c \frac{C_{*} h}{h+\varepsilon}}+C \ell_{h}+I_{2} C \sum_{j}\left(h / d_{j}\right)^{r-1-s} .
$$

Since $r-1-s>1$, choosing $C_{*}$ once again large enough, we can conclude that

$$
I_{2} \leq C C_{*}^{N / 2+s} e^{c \frac{C * h}{h+\varepsilon}}+C \ell_{h} .
$$

Thus the proof of Proposition 6.2 is complete.

\section{Appendix. Proof of lemma 2.2}

Proof. To show the estimates for $K^{\varepsilon}(x, y)$, we use the Green's function $G(x, y ; t)$ for the parabolic problem

$$
\begin{aligned}
G_{t}(x, y ; t)-\Delta G(x, y ; t) & =0 \quad \text { in } \Omega, t>0, \\
\frac{\partial G(x, y ; t)}{\partial n} & =0 \quad \text { on } \partial \Omega, \\
G(x, y ; 0) & =\delta_{x}(y) .
\end{aligned}
$$

Since $u$ satisfies

$$
\begin{aligned}
-\Delta u+\frac{u}{\varepsilon^{2}} & =\frac{f}{\varepsilon^{2}}, \quad \text { in } \Omega, \\
\frac{\partial u}{\partial n} & =0, \quad \text { on } \partial \Omega,
\end{aligned}
$$

by the Theorem 4 in $[\underline{3}$, we have the following representation:

$$
u(x)=\int_{\Omega}\left[\int_{0}^{\infty} e^{-\frac{z}{\varepsilon^{2}}} G(x, y ; z) d z\right] \frac{f(y)}{\varepsilon^{2}} d y,
$$

where $G$ is the Green's function for the parabolic problem. With a change of variables $t=z / \varepsilon^{2}$, we obtain

$$
\varepsilon^{-2} \int_{0}^{\infty} e^{-\frac{z}{\varepsilon^{2}}} G(x, y ; z) d z=\int_{0}^{\infty} e^{-t} G\left(x, y ; \varepsilon^{2} t\right) d t .
$$


Define

$$
K^{\varepsilon}(x, y)=\int_{0}^{\infty} e^{-t} G\left(x, y ; \varepsilon^{2} t\right) d t
$$

Thus we have the following representation:

$$
u(x)=\int_{\Omega} K^{\varepsilon}(x, y) f(y) d y .
$$

Since the coefficients in the parabolic equation (7.1) are time independent, we have the following estimate for the parabolic Green's function.

Lemma 7.1. Assume that $\partial \Omega$ in problem (7.1) is sufficiently smooth. Then for any multi-index $m$ there exist constants $c_{2}, c_{3}, C$ such that for $0<t<\infty$,

$$
\left|D_{x}^{m} G(x, y ; t)\right| \leq C t^{-\frac{N+|m|}{2}} e^{c_{2} t-c_{3} \frac{|x-y|^{2}}{t}} .
$$

The proof of this result can be found in 3], Theorem 3 in particular.

Using Lemma 7.1, we have

$$
\left|D_{x}^{m} K^{\varepsilon}(x, y)\right| \leq C \int_{0}^{\infty} \frac{e^{-t\left(1-\varepsilon^{2} c_{2}\right)-c_{3} \frac{|x-y|^{2}}{\varepsilon^{2} t}}}{\left(\varepsilon^{2} t\right)^{\frac{N+|m|}{2}}} d t .
$$

To estimate this integral we use the following lemma.

Lemma 7.2. There exist constants $C$ and $c_{0}$ independent of $d$ such that

$$
\int_{0}^{\infty} \frac{e^{-c_{4} t-c_{3} \frac{d^{2}}{t}}}{t^{M / 2}} d t \leq C e^{-c_{0} d} \begin{cases}1, & \text { if } M=1, \\ 1+|\log d|, & \text { if } M=2, \\ d^{2-M}, & \text { if } M>2 .\end{cases}
$$

Proof. The proof is adapted from [3. First we split the integral into two parts.

$$
\int_{0}^{\infty} \frac{e^{-c_{4} t-c_{3} \frac{d^{2}}{t}}}{t^{M / 2}} d t=\int_{0}^{1} \frac{e^{-c_{4} t-c_{3} \frac{d^{2}}{t}}}{t^{M / 2}} d t+\int_{1}^{\infty} \frac{e^{-c_{4} t-c_{3} \frac{d^{2}}{t}}}{t^{M / 2}} d t=I_{1}+I_{2} .
$$

In order to estimate $I_{1}$, we consider two cases, $d \leq 1$ and $d>1$ :

Case 1: $d \leq 1$,

$$
I_{1} \leq \int_{0}^{1} \frac{e^{-c_{3} \frac{d^{2}}{t}}}{t^{M / 2}} d t
$$

For $M>2$, by making a change of variables $z=\frac{d}{\sqrt{t}}$, we have

$$
I_{1} \leq \frac{2}{d^{M-2}} \int_{d}^{\infty} e^{-c_{3} z^{2}} z^{M-3} d z \leq \frac{C}{d^{M-2}} .
$$

For $M=2$ by letting $z=c_{3} d^{2}$ and making a change of variables $w=\frac{z}{t}$, we have

$$
I_{1} \leq \int_{0}^{1} \frac{e^{-\frac{z}{t}}}{t} d t=\int_{z}^{\infty} \frac{e^{-w}}{w} d w \leq\left|\int_{z}^{1} \frac{d w}{w}\right|+\int_{1}^{\infty} e^{-w} d w=|\log z|+e^{-1} .
$$

Finally for $M=1$,

Case 2: $d>1$,

$$
I_{1} \leq \int_{0}^{1} \frac{e^{-c_{3} \frac{d^{2}}{t}}}{\sqrt{t}} d t \leq \int_{0}^{1} \frac{1}{\sqrt{t}} d t=2
$$

$$
I_{1}=\int_{0}^{1} \frac{e^{-c_{4} t-c_{3} \frac{d^{2}}{t}}}{t^{M / 2}} d t=\int_{0}^{1} \frac{e^{-c_{4} t-c_{3} \frac{d^{2}}{2 t}} e^{-c_{3} \frac{d^{2}}{2 t}}}{t^{M / 2}} d t .
$$


The function $-c_{4} t-\frac{c_{3} d^{2}}{2 t}$ has a maximum at $t=d \sqrt{\frac{c_{4}}{2 c_{3}}}$ equal to $-d \sqrt{2 c_{4} c_{3}}$, and the function $e^{-c_{3} \frac{d^{2}}{2 t}} t^{-M / 2}$ has a maximum at $t=\frac{2 c_{3} d^{2}}{M}$ equal to $e^{-M / 2}\left(\frac{M}{2 c_{3} d^{2}}\right)^{M / 2}$. Thus,

$$
I_{1} \leq e^{-d \sqrt{2 c_{4} c_{3}}} \int_{0}^{1} \frac{e^{-c_{3} \frac{d^{2}}{2 t}}}{t^{M / 2}} d t \leq C e^{-d \sqrt{2 c_{4} c_{3}}} .
$$

Now we estimate $I_{2}$ for any $d>0$. We have

$$
I_{2}=\int_{1}^{\infty} \frac{e^{-c_{4} t-c_{3} \frac{d^{2}}{t}}}{t^{M / 2}} d t=\int_{1}^{\infty} \frac{e^{-\frac{c_{4} t}{2}-c_{3} \frac{d^{2}}{t}} e^{-\frac{c_{4} t}{2}}}{t^{M / 2}} d t
$$

Again using that $-\frac{c_{4} t}{2}-\frac{c_{3} d^{2}}{t}$ has a maximum at $t=d \sqrt{\frac{2 c_{4}}{c_{3}}}$ equal to $-2 d \sqrt{2 c_{4} c_{3}}$, we have

$$
I_{2} \leq e^{-2 d \sqrt{2 c_{4} c_{3}}} \int_{1}^{\infty} \frac{e^{-\frac{c_{4} t}{2}}}{t^{M / 2}} d t \leq C e^{-2 d \sqrt{2 c_{4} c_{3}}}
$$

and the proof of Lemma 7.2 is complete.

Provided that $1-\varepsilon^{2} c_{2}>0$, we apply the previous lemma with $d=\frac{|x-y|}{\varepsilon}$ and $c_{4}=1-\varepsilon^{2} c_{2}$, to conclude the proof of Lemma 2.2.

\section{ACKNOWLEDGMENTS}

The author's research was supported by an NSF VIGRE Fellowship. The author is grateful to Alfred Schatz, Lars Wahlbin, and Timothy Warburton for interesting and valuable discussions. The author would also like to thank the anonymous reviewer for very insightful comments and for helping to improve the presentation of the paper.

\section{REFERENCES}

1. I.A. Blatov, On the Galerkin finite-element method for elliptic quasilinear singularly perturbed boundary value problems. I. (in Russian) Differentsial'nye Uravneniya 28 (1992), no. 7, 11681177; translation in Differential Equations 28 (1992), no. 7, 931-940. MR.1201213(94a:65056)

2. C. Clavero, J.L. Gracia, and E. O'Riordan, A parameter robust numerical method for a two dimensional reaction-diffusion problem, Math. Comp., posted on June 7, 2005, PII S 00255718(05)01762-X (to appear in print). MR2164094(2006e:65192)

3. S.D. Ėı̆del'man and S.D. Ivasišen, Investigation of the Green's matrix for a homogeneous parabolic boundary value problem, Trans. Moscow Math. Soc. 23 (1970), 179-242. MR0367455 (51:3697)

4. W. Eckhaus, Asymptotic Analysis of Singular Perturbations, North-Holland, Amsterdam, 1979. MR.553107 (81a:34048)

5. N. Kopteva, Maximum norm error analysis of a $2 d$ singularly perturbed semilinear reactiondiffusion problem, to appear in Math. Comp.

6. J.P. Krasovskii, Properties of Green's function and generalized solutions of elliptic boundary value problems, Soviet Mathematics (Translations of Doklady Academy of Sciences of the USSR) $10^{2}$ (1969), 54-120.

7. A.H. Schatz, Pointwise error estimates and asymptotic error expansion inequalities for the finite element method on irregular grids: Part 1, Math. Comp. 67 (1998), 877-899. MR.1464148 (98j:65082)

8. A.H. Schatz, V. Thomée, and L.B. Wahlbin, Stability, analyticity, and almost best approximation in maximum-norm for parabolic finite element equations, Comm. Pure Appl. Math. 51 (1998), 1349-1385. MR:1639143(99h:65171) 
9. A.H. Schatz and L.B. Wahlbin, Interior maximum norm estimates for finite element methods, Math. Comp.31 (1977), 414-442. MR0431753 (55:4748)

10. A.H. Schatz and L.B. Wahlbin, On the quasi-optimality in $L_{\infty}$ of the $\stackrel{\circ}{H^{1}}$-projection into finite element spaces, Math. Comp.38 (1982), 1-22. MR637283 (82m:65106)

11. A.H. Schatz and L.B. Wahlbin, On the finite element method for singularly preturbed reactiondiffusion problems in two and one dimensions, Math. Comp. 40 (1983), 47-89. MR679434 (84c:65137)

12. V. Thomée and L.B. Wahlbin, Maximum-norm estimates for finite-element methods for a strongly damped wave equation, BIT 44 (2004), 165-179. MR2057368 (2005g:65151)

13. L.B. Wahlbin, Local behavior in finite element methods, Handbook of Numerical Analysis, Vol II, Finite Element Methods (Part 1), P.G. Ciarlet and J.L. Lions, Eds, Elsevier, 1991, 355-522. MR:1115238

Department of Computational and Applied Mathematics, Rice University, Houston, TEXas 77005

E-mail address: dmitriy@caam.rice.edu 